\section{GLOBAL CHILD HEALTH}

Antibiotics in non-bloody diarrhoea

Managing infectious diarrhoea is essentially uncomplicated. In only a small proportion of children, those with bloody diarrhoea (shigella, cholera amoebiasis) and associated toxicity or sepsis are antibiotics indicated. In other situations, they can prolong the illness and complicate recovery. We know the practice continues, but to date, there has been little data on its extent which is why Auta's meta-analysis of routinely available Demographic Health Survey (DHS) data from a number of Sub Saharan African Countries is so welcome. Using a random effects model to allow for intercountry heterogeneity, they estimated that $23.1 \%$ (95\% CI $19.5 \%$ to $26.7 \%$ ) of children under 5 years old with non-bloody diarrhoea were prescribed antibiotics. Family wealth and higher maternal education was associated with higher rates of antibiotic treatment and, in broad terms, children in western Africa were more likely to be treated than those in the East, differences that probably reflect long standing parental expectation and medical practices. See page 518 .

\section{ACUTE LYMPHOBLASTIC LEUKAEMIA TREATMENT COMPLETION IN CHINA}

Until 2003, most children with ALL in China either failed to start or abandoned treatment, financial restrictions being the the most common reason. That year marked the start of a major demographic and economic transition (in the aftermath of the severe acute respiratory syndrome (SARS) epidemic) with an escalation in government funding of health. Since 2010, the New Rural Cooperative Medical Scheme has covered the majority of the treatment costs of ALL, a change that has coincided with the inception of paediatric oncology units and standardisation of guidance. Tang and colleagues assessed rates of treatment completion between

Department of Women's and Children's Health, International Maternal and Child Health (IMCH), Uppsala University, Uppsala, Sweden; Department of Paediatrics, Länssjukhuset Gävle-Sandviken, Gävle, Sweden; Department of Child Health, Aga Khan University, Karachi, Pakistan

Correspondence to Dr Nick Brown, Department of Women's and Children's Health, International Maternal and Child Health (IMCH), Uppsala University, Uppsala 75237, Sweden; nickjwbrown@gmail.com
2015 and 2016 and quantitative and qualitative predictors of abandonment in children treated in 20 major hospitals across 10 provinces. The findings were hugely impressive with a 3 year survival rate of 93\% and cumulative abandonment rate of just $2.8 \%$ at 50 weeks. Abandonment occurred predominantly during induction and was associated with low income region of descent, higher risk disease and (in the interviews) a perception by some parents, of incurability, these now though only a small minority. See page 522.

\section{OUT OF HOSPITAL CARDIOPULMONARY RESUSCITATION}

Improved skill in CPR for lone lay providers unequivocally enhances chances of survival from out of hospital cardiac arrest: this is one of the catalysts for the 'Kids Save Lives' and 'Restart a Heart' initiatives. What is less clear is whether a two finger or two thumb chest-encircling approach is more effective in infant resuscitation in terms of provision of the prerequisite rate and depth, allowance of recoil and avoidance of overventilation. Pellegrino tested the methods directly against each other in a group of young adults (median age 17 years) recruited from US schools and community services using a manikin-based randomised posttraining crossover trial. The overall differences were small, but the two thumb method resulted in a mean better chest compression depth and, importantly, was more popular. The implications in terms of training are contextualised by Gregson's editorial. See pages 530 and 511 .

\section{SOURCE OF FEVER}

Despite the evolution of discriminators over time from clinical absolute neutrophil counts to acute phase markers (CRP and procalcitonin particularly) differentiating viral from bacterial fever in infants is still difficult. Gomez measured the performance of a combination of predictors in 196 infants aged under 3 weeks and 1331 between 3 weeks and 3 months of age presenting to a tertiary paediatric emergency department in Spain. They found disappointingly low predictive values with a sensitivity and negative likelihood ratio for ruling out IBI in the younger group with values of $44.4 \%$ (95\% CI $18.9 \%$ to
Nick Brown ${ }^{\circledR}$, Editor in Chief

$73.3 \%$ ) and 0.79 (95\% CI 0.43 to 1.44 ), respectively. The test performed much better in the older children with a sensitivity of $84.6 \%$ (95\% CI $57.8 \%$ to $95.7 \%$ ) and NPV of 0.19 (95\% CI 0.05 to 0.67$)$. The advent of blood and CSF viral PCR testing should now mean shorter antibiotic courses but, in other respects we are much as we were in terms of immediate decision making: we're neither good at ruling bacterial sepsis in or out in the acute situation. See page 547.

\section{BODY COMPOSITION}

The limitations of BMI is a measure of body fat are well known: it is unable to distinguish between fat and fat-free mass, an important problem when used as a marker at both an individual and population level as a predictor of cardiovascular risk. The tri-ponderal index (TMI), calculated by mass $/$ height $^{3}$ has been with us for a number of years. Proponents cite its statistical stability as a reason for which to moot it as a replacement even though there is little evidence to corroborate this assumption. To test this hypothesis, Kuhle and colleagues analysed data from the Canadian Health Measures Survey and modelled the predictive value of TMI in children aged 6-19 years of age. They derived centiles for TMI and ROC curves, testing these against BMI, but, found no notable difference in AUC values for the two models in terms of standard cardiovascular risk markers. This surprising negative result marks another chapter in the chequered career of anthropometric measurement and perhaps reopens the door to other candidates like bioimpedance. See page 577.

\section{MEDICATION SAFETY}

The paper by Kahn and Abramson in the Quality Improvement section gives us an update of recent innovations. Improved (safer) prescribing is possible through electronic systems, decision support systems, unit based pharmacists, apps and workflow management. Systems to improve administration include prescriptions matched by barcodes to patients and smart pumps. The paper is put into perspective in the podcast (on our website) by Claire Lemer and Dan Hawcutt. See page 596. 